\title{
The Analysis of the Impact of Yoga on Healthcare and Conventional Strategies for Human Pose Recognition
}

\author{
Nagalakshmi Vallabhanenia ${ }^{a}$ Dr. P. Prabhavathy ${ }^{b}$ \\ aResearch Scholar, School of Information Technology \& Engineering, VIT Vellore, TamilNadu, India \\ E-mail: nagalakshmi.2019@vitstudent.ac.in \\ ${ }^{\mathrm{b}}$ Associate Professor, School of Information Technology \& Engineering, VIT Vellore, TamilNadu, India \\ E-mail: pprabhavathy@vit.ac.in
}

Article History: Received: 10 November 2020; Revised 12 January 2021 Accepted: 27 January 2021; Published online: 5 April 2021

\begin{abstract}
Human pose estimation is a profound, established computer vision issue that has uncovered numerous past difficulties. Breaking down human exercise is advantageous in multiple fields like surveillance, biometrics, and many healthcare applications. Workout with yoga poses is famous these days since yoga activities can expand adaptability and muscular quality, and the respiration procedure will be improvised. The yoga postures evaluation is hard to check, so specialists will most likely be unable to benefit from the exercises ultimately. IoT-based yoga frameworks are required for individuals who need to rehearse Yoga at home. A few studies are recommended camera-oriented or wearable gadget-oriented yoga posture finding strategies with more precision. Nonetheless, camera-based plans have security and privacy issues, and the wearable device-based methods are illogical in the earlier applications. To build such systems, one must have a strong foundation and current research in pose estimation. In this paper, first, the impact of Yoga on humans with various stress levels is analysed on the real-time data. Second, the comprehensive review of yoga posture recognition systems from machine learning to deep learning strategies and evaluation metrics discussed
\end{abstract}

Keywords: pose estimation, yoga pose recognition, activity recognition, IoT, deep learning

\section{Introduction}

Yoga started in India many decades ago, gathering exercise related to mental, physical, and profound strength. Yoga and sports have been drawing in people groups for endless years, yet from the most recent decade, an enormous community accepts Yoga as a feature of their life. The reason is because of the medical advantages. It is critical to do this activity in the right manner, particularly in the right pose. At some point, because of no assistance or information, individuals don't have a better idea about the correct technique to perform Yoga and begin doing Yoga with no proper guidance. Accordingly, they harm them-self while self-preparing because of an ill-advised stance. Yoga ought to perform under the direction of a trainer, yet it is likewise not moderate for all individuals. These days individuals utilize their cell phones to figure out how to do yoga stances and begin doing that, yet while doing that, they don't realize that the yoga present they are doing is precisely or not. To conquer the constraints, as mentioned earlier, numerous works have been proposed.

Computer vision and data science methods are utilized Artificial Intelligence (AI) products that act as a trainer. It depicts the benefits of that present. In some articles, there applied AI and Deep learning modules on an enormous number of picture dataset, which includes different yoga poses (Laxman, 2020). Yoga utilizes a progression of physical postures called asana, breathing control, and meditation. Since Yoga focuses on both body and brain, it is unmistakably more remedial than work out. Yoga practice must consolidate the extending of significant muscle gatherings, adding to physical ability and adaptability (Rowland et.al, 2020). A few yogabased mediations directed in the community have been described. Regardless of how interventions assist in decreasing falls and the dread of losing, older people with these issues may not promptly approach such networkdependent projects. Particular old individuals manifest inconvenience at rehearsing Yoga publicly, which is particularly valid for ladies who want to do much at home and recreation. It is desirable to train Yoga in a bounded area with no disturbance. In rural zones, notwithstanding, yoga coaches are scarce (Mohan, 2021), and this factor is a significant driver in the production of a self-helped yoga training framework. Learning is frequently connected with two criteria, area and time, and is relevant to exercise or Yoga by the older too. Given an overall hesitance concerning the old to exercise or practice Yoga, the spot and season of training are adaptable by their inclinations. Also, learning depends on the person's choices and inspiration to learn at their movement. 
Alongside the advantages such as improved equalization, Yoga has a disadvantage. It tends to be rehearsed at home, in a composed setting, alone, or a gathering. Ideally, Yoga fits self-learning, too (Wang et.al, 2020). Yoga includes various successions of stances, which are assorted positions a person can expect. In Yoga, the exact posture is basic. The posture successions accepted during training are recognized and dissected to plan a selfpreparing yoga framework, and wrong postures are evaluated.

Due to advancements in deep neural networks, estimation of the pose has attained the best performance (Kothari, 2020 \& Mathis, 2020). The nature of the central issue and skeleton comments in these datasets assume a significant part in achieving the state-of-the-art assessment models. Nonetheless, the manual explanation measure is inclined to human blunders and can be seriously influenced by different factors, for example, goal, impediment, light, viewpoint, and variety of stances (Palanimeera, 2020). For instance, Figure 1 represents some human posture pictures from the Yoga-82 dataset (Verma. et.al, 2020) for the yoga movement, which characteristically comprise probably the most different means that the human body can perform. It may be seen that a portion of these stances is too hard to even think about being caught from one perspective. This turns out to be more troublesome with the adjustments in image resolution and impediments. These elements deliver subtle posture comments. For example, significant points and skeletons for the restricted objects in these pictures may be impossible as it will prompt false and complicated explanations. The remaining part of the paper is structured as follows. Under section 2, the need and impact of Yoga on healthcare on the real-time data are described. In section 3, the human pose estimation and the background methodology addressed. Section depicts a comprehensive review of posture recognition techniques, along with evaluation metrics, is presented. Section 5 concludes the paper.

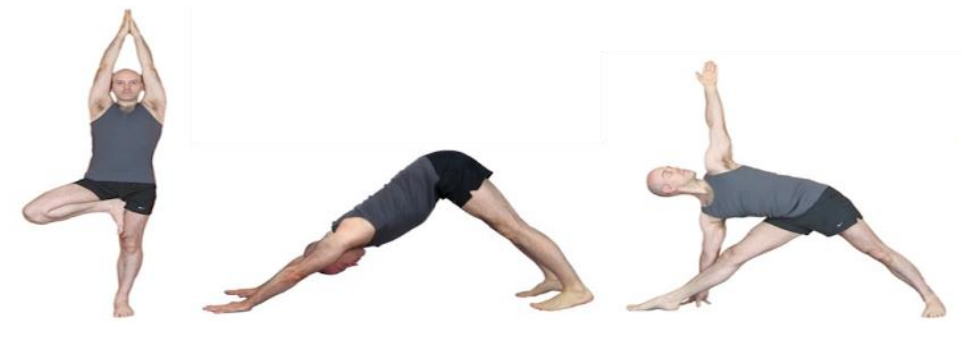

a
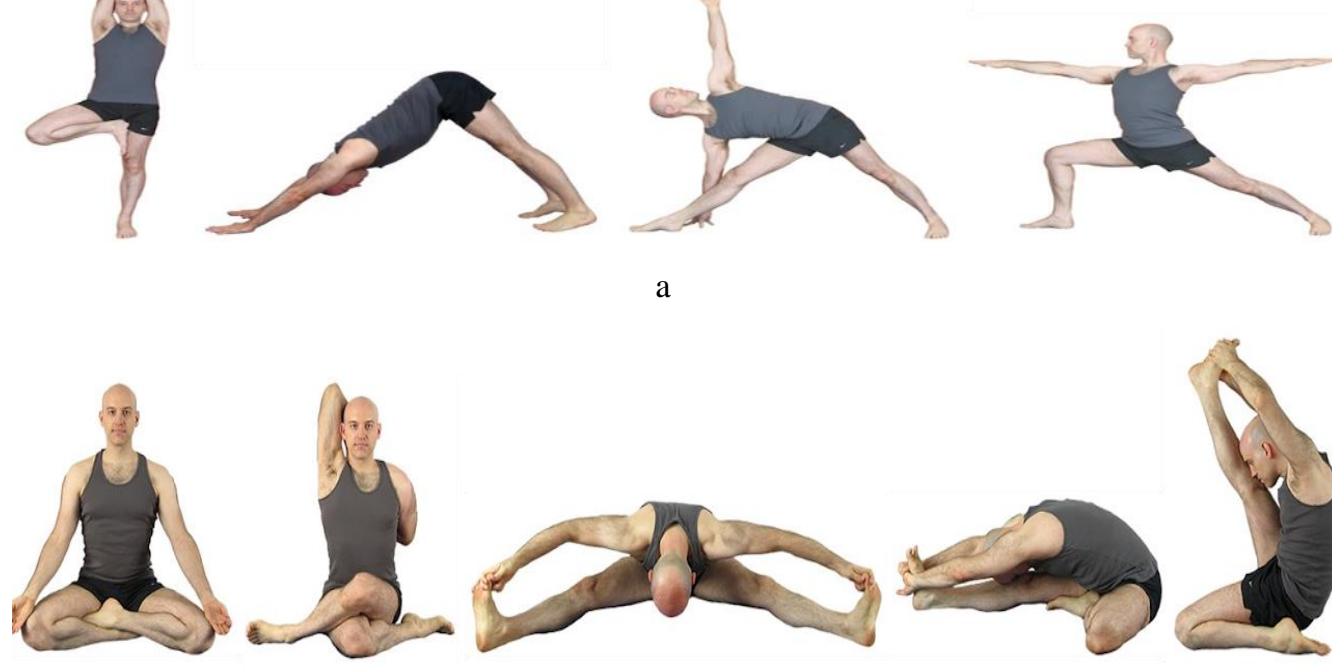

b
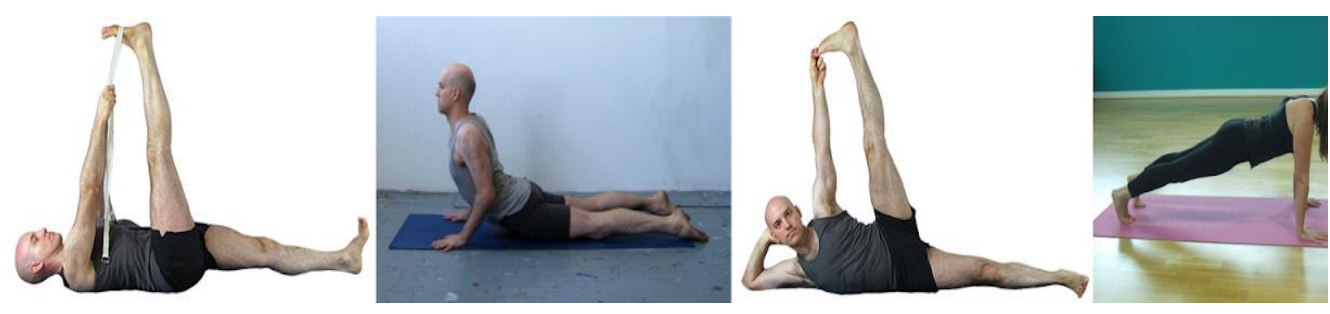

$\mathrm{c}$

Figure 1: Sample images from the dataset (Yoga-82). a. standing, b. sitting, c. reclining.

\section{Need and Impact of Yoga on healthcare}

The COVID outbreak and resulting lockdown had constrained us all to stay at homes prompting a more unpleasant life (Borave, 2020). Public distancing had invigorated the chance to improve mental ability and Pranayama and Meditation (Bilmez, 2020). Yoga is perhaps the most straightforward physical, mental, and Spiritual work during this isolation period. "Yoga Practice deals with these psychological issues, and along with 
breathing, meditation is the best general practice which will be careful with our body, brain, and soul. Yoga can help lessen pressure and uneasiness and aids in improving the physical, just as assuaging negative mental impacts. Furthermore, it encourages you to plan your day better during lockdown time. The fundamental point of Yoga in our everyday life is to create Physical, Mental, Social, and Spiritual well-being (Rajendar, Mala et.al, 2020). Yoga's impact on the people in the lockdown period is analysed. The data collected from 109 persons, including 64 persons who are male and 45 are female. We have raised a few questions to check the health issuelevel and the respondents' subsequent answers. Further, five parameters are considered: stress level, peace of mind, consciousness, physical health, and the respondent's mental health. The analyzed results are shown in Figure 2 to Figure 4.

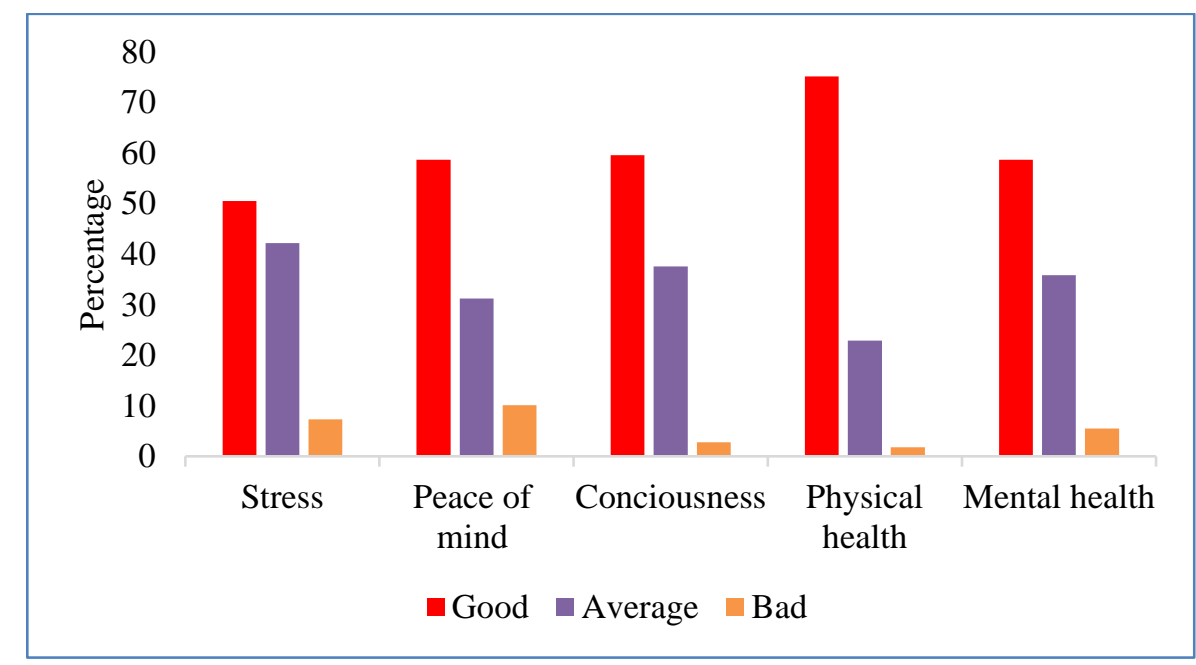

Figure 2: Health issue levels during lockdown by different respondents

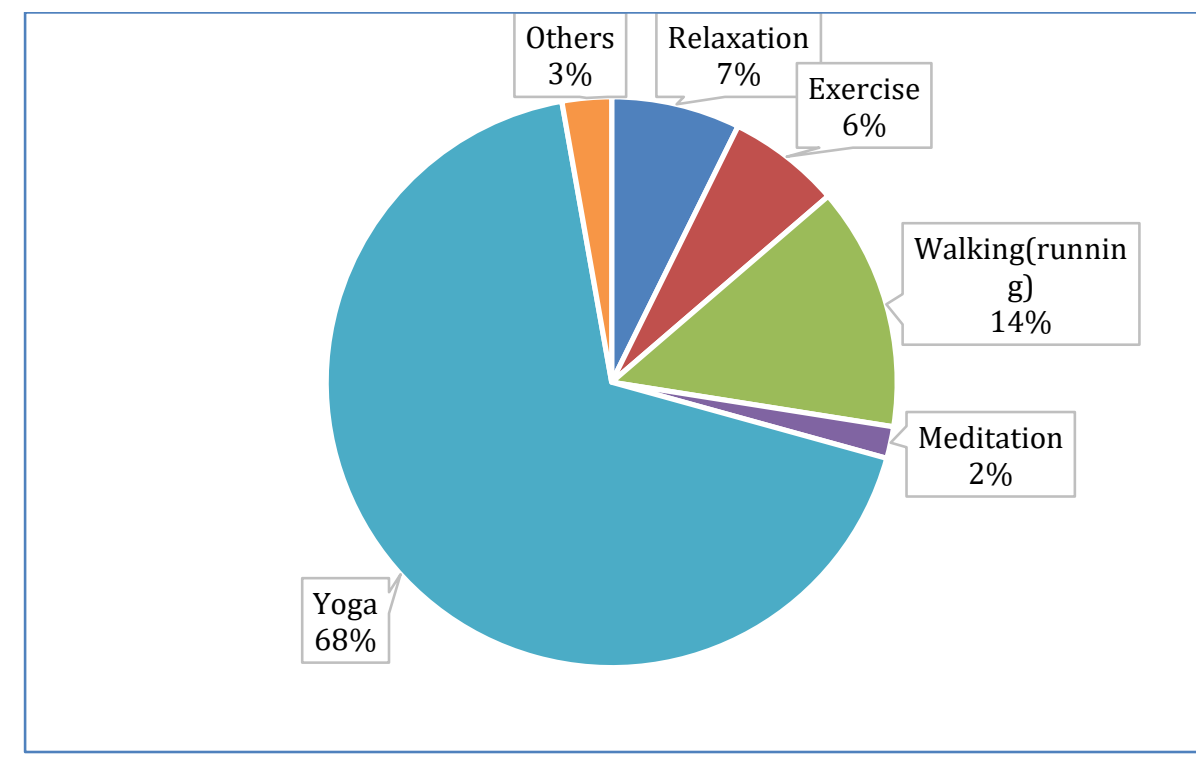

Figure 3: Health management technique used by different respondents.

The respondents' stress levels in the lockdown period are about $50.5 \%$ good, $42.2 \%$ average, and $7.3 \%$ bad. Further, peace of mind is $58.7 \%$ good, $31.2 \%$ average, and $10.1 \%$. The consciousness includes $59.6 \%$ good, $37.6 \%$ average, and $2.8 \%$ bad. These populations' physical health includes $75.2 \%$ good, $22.9 \%$ average, and $1.8 \%$ bad, and mental health has $58.7 \%$ good, $35.8 \%$ average, and $5.5 \%$ bad. The other factors that $15.6 \%$ of the population opted to stress levels increased, and $12.8 \%$ of people opted for frustration increased, $5.5 \%$ opted depression increased, and $14.7 \%$ opted concentration decreased. Further, we have also collected the health reasons mentioned earlier, among which many respondents opted for financial, professional, and personal reasons. Among these, $67.9 \%$ of respondents opted for Yoga as a health management technique, and $90.8 \%$ of people opted for Yoga's positive impact on better health. 


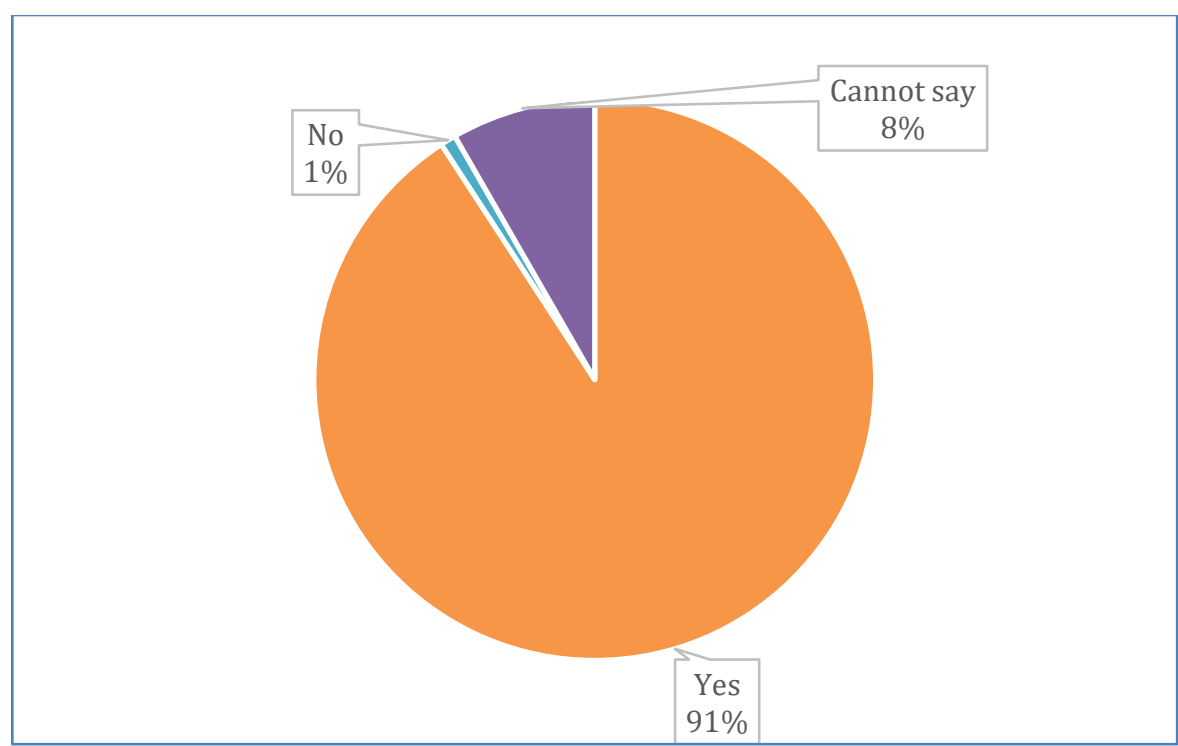

Figure 4: Positive impact of Yoga on health by different respondents

\section{Human Pose Estimation}

Human Pose Estimation (HPE) is an essential computer vision activity highly preferred in the research domain. It is also performed in various applications like recognition of activities, humanity, movies, animation, the interaction between humans and computers, and so on (Fourie, 2020 \& Jain, 2020).

Movies and animation: Generating many key digital characters is a contrast from capturing human activities. Modest and exact human movement catch framework can enhance the advancement in the computerized movie industry.

Virtual reality: The highly challenging technology which could be executed in both academic and entertainment domains. The social stance assessment can also explain the association among human and computer-generated reality worlds and upgrade the intuitive experience.

Human-computer interaction (HCI): It is majorly chosen for computers and also for robots to analyse recognition, place, and human movement in a better way. With the human pose, computers and robots could run the code straightforward and be highly intelligent.

Video surveillance: It adopts HPE advancement during tracking, recognition of activity, and humans' reidentification in a specified range.

Medical assistance: In clinical help, HPE can furnish doctors with quantitative human movement data, particularly for recovery and physical treatment.

Sports motion analysis: Assessing players' stance in sports recordings can also acquire competitors' pointers (for example, running, jumping). While training, HPE could give a quantitative examination of activity data. In physical exercise, educators focus on the assessments of students with HPE. Monocular human posture assessment has some attractive attributes and difficulties. The difficulties of human posture assessment majorly fall into three viewpoints.

(1). Flexible bodies demonstrate complex reliant joints and a great extent of opportunity, which may cause self-impediments or uncommon/problematic stances.

(2). numerous body appearances incorporate changed attire and self-comparative parts.

(3). The critical environment might lead to foreground occlusion of neighbors' same parts, viewing many angles, and camera view truncation.

\section{1 Categories of HPE}

This segment sums up the various classifications of deep learning-dependent HPE strategies based on multiple attributes. Two of the commonly used categories are: 1) generative and discriminative. 2) top-down and bottom-up. 3) Regression-based and recognition-based methods. 4) one-stage and multi-stage.

Generative Vs. Discriminative: Major discrimination among generative and discriminative strategies is the utilization of body models. Considering multiple descriptions of the human body models, the generative approaches could be applied in various ways, likely prior perceptions regarding body contour maps. Discriminative approaches straightforwardly study how to map between input sources and human posture space (learning-based) otherwise find from available instances (instance-based) with no human body patterns involved. 
Top-down Vs. Bottom-up: Multi pose estimation includes HPE techniques and is categorized into a top-down approach and bottom-up approach as per the prediction's starting point: high-level abstraction or low-level pixel evidence. Former approaches initiate from high-level abstraction to first identify persons and form person place in existing boxes. On the other hand, bottom-up approaches initially predict each person's body parts in an input image and later integrate them through human body model fitting or different paradigms. Notice that the body parts are joints, limbs, or small template patches that rely on various approaches. Nevertheless, if people with huge overlap exist, bottom-up approaches face the challenges of including the body's corresponding parts.

Regression-based Vs. Recognition-based: Considering various issue formulations, deep learning-dependent human posture estimation procedures are classified under regression-oriented or detection-related approaches. Former methods straightforwardly link input images to the coordinates of body joints or human body models' parameters. The detection-based methods consider the body parts as detection targets based on two extensively utilized representations: image patches and heat maps of joint locations. In contrast to actual image size, the obtained outputs of small-region representation restrict the end joint coordinates' preciseness.

One-stage Vs. Multi-stage: The deep learning-based one-stage mechanisms concentrate on mapping an input image to the human postures using end-to-end networks. Multi-stage techniques forecast human posture in many phases and are accompanied by intermediate supervision.

\subsection{Human Body models}

Human body modeling is an essential element of HPE. The human body is a flexible and complex non-rigid object and contains particular features such as the kinematic body, body contour, surface texture, etc. We consider different representation levels and application scenarios, as represented in Figure 5.

Skeleton-based Model: This model is otherwise called a stick-figure or kinematic model. It depicts a group of joints (typically 10-20) and the relative limb orientations following the human body skeletal structure. The skeleton-based model can also be depicted as a graph in which the vertices represent joints and edges, encoding constraints or prior connections of joints in the skeleton structure. This human body's topology is flexible and straightforward, which can be extensively utilized in both 2D and 3D HPE (Cao. et.al, 2017 \&Mehta, 2017) and human pose datasets (Wu.et.al, 2017). With the obvious benefits, this representation has several shortcomings, like lacking textual data indicating no width and contour data of the human body.

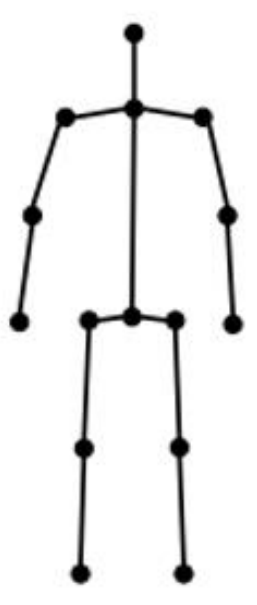

a

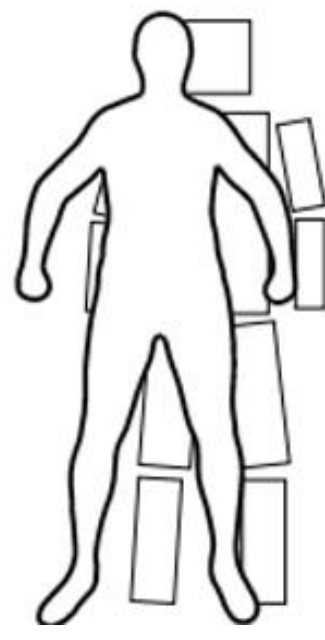

b

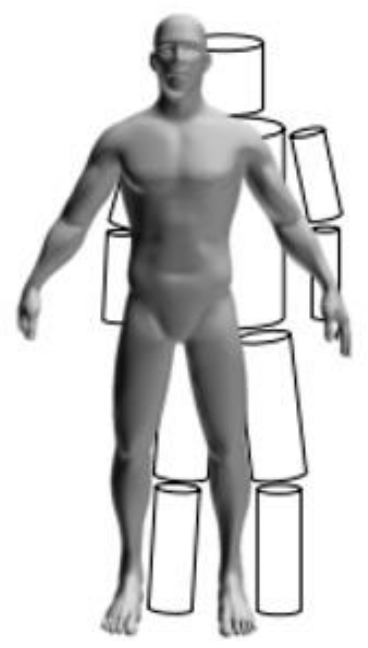

$\mathrm{c}$

Figure 5: Human body models. a. skeleton-based, b. contour-based, c. volume-based models

Contour-based Model: This can be frequently utilized in earlier HPE techniques having the rough width and contour data of body limbs and torso. Parts of the human body are relatively represented with the rectangles or boundaries of a person's silhouette (Ju et.al, 1996 \& Chi N.K, 2011).

Volume-based Model: 3D shapes and poses of the human body are usually denoted by volume-based patterns with geometric structures or meshes. Prior geometric representations for modeling the body parts contain cylinders, conics, and so on. Modern volume-based models are denoted in mesh format, usually captured with 3D scans. Extensively applied volume-based models include Shape (Looper et.al, 2015 \& Joo et.al, 2018). 


\section{Literature Review}

Human posture estimation has been a significant issue in computer vision, and numerous benchmark datasets are introduced previously. In (Ding et.al, 2020), a posture recognition technique using many characteristics and rule learning is applied. According to such a method, the features are first obtained from the dataset, and rule learning is applied to the features, and classification is applied. The rule learning includes the bagging approach, and the methodology of the bagging process is shown in Figure 6. Practicing Yoga can help persons to overcome depression, insomnia, anxiety, and so on. Due to Yoga's many advantages, there should be more self-training systems (Marinez , 2017).

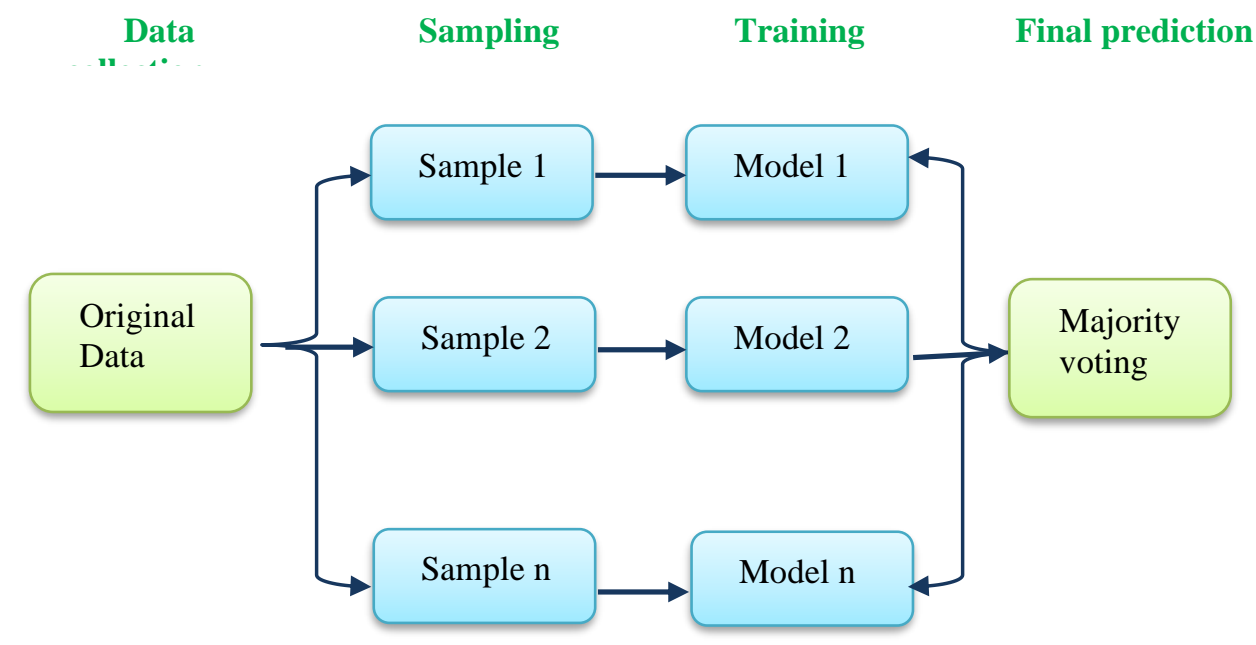

Figure 6: Procedure of bagging approach (Liu, H, 2015).

Researches concentrated on yoga posture identification and rectification (Islam et.al, 2017). Few authors have utilized a Kinect gadget to make a human pose. This gadget is being used for capturing pictures. The critical portion is that this gadget includes an inbuilt infrared laser projector, a multi-array microphone, and an RBG camera for color capturing and depth images. This gadget also has an instrument to show a human body skeleton in 3D space that provides the body joint coordinates' details. This technique is good, but this approach's major drawback is that this Kinect gadget is cost-effective and not user-friendly. To prevent this issue, the tf-pose paradigm is applied. This paradigm forms a human body's skeleton and provides essential data regarding the human body's joint. Pullen, P., \& Seffens, W. (2018) utilized the Kinect sensor's visual Gesture Builder feature to snap the Yoga poses with more precision. Edwin W. Trejo, Peijiang Yuan (2018) utilized Microsoft Kinect v2, which yields high accuracy and precision, but it needs much computational time for a highly complicated model. The Kinect camera works with three features (depth, color, and body tracking). With all these features of the Kinect gadget, those generated a Computer Interaction framework to train an Adaboost paradigm that o detects six common Yoga poses. Another approach for pose recognition for utilizing a Convolution Neural Network.

Furthermore, researchers applied six yoga asanas datasets. They utilized a deep learning model of CNN and LSTM to detect a yoga posture where the features are retrieved by using CNN from the points obtained through Open Pose, and LSTM is utilized to identify Yoga's posture. By this module, those can attain $99.04 \%$ accuracy over a frame. Chen et al. (2013) recommended a self-training framework to perform Yoga properly and avoid injuries with lewd poses. Patil et al. (2011) depicted "Yoga tutor" with the Speeded Up Robust Features (SURF) so that to find and show to perform asana pose among the practitioner and the trainer. Though, just the shape data acquired from one specific way can't portray and separate the asana execution properly. Luo et al. (2011) represented a Yoga preparing framework dependent on the Movement Replication method (MoRep). The developed Interface Suit, comprising of Inertial Measurement Units (IMUs) and tactors, catches body movements; however, it impacts the professional's working out (Wu, W, 2010). Chen et al. (2014) discovered a yoga posture recognition mechanism for self-training, which employed a Kinect camera. They gathered more than 290 snaps of twelve yoga asanas performed by five yoga practitioners, with every pose performed five times. Initially, the body shape is retrieved from those snaps; later, a star skeleton is applied to describe Yoga's postures. The accuracy of the system was more than 99.33 percent. Wu et al. (2011) recommended a remote interactive yoga learning/training system via the Internet utilizing Webcam and image detection. The plan identifies posture while performing Yoga by contrasting the transformation distance between the human 
silhouette and the base facts. They gathered more than 400 videos in which six persons performed every three videos for every pose and 23 postures. The computer provides the score ranges between 0 and 100 for the detected posture, and nearly 86 percent of the difference between computer calculated scores and the yoga trainer scores lies between -2.5 to 2.5 . Besides, Islam et al. (2017) introduced a yoga pose detection system that leverages 15 joint human points from Kinect camera images. Likely, Wang et al. (2016) proposed a model to find pose employing Kinect camera. They retrieved human silhouettes and used a learning vector quantization neural network for five fundamental pose detection. The accuracy of the system was greater than 97 percent. However, these pose detection frameworks yield more recognition rates, so those are treated as privacy-invasive. Yao et al. [35] represented an unobtrusive human pose recognition strategy employing a passive RFID signal. The method identifies the human poses considering the RSSI signal analysis generated during a person performing the asana in the middle of an RFID tag array and an RFID antenna. This system attains an accuracy of 99 percent for 12 poses.

Kasman and Moshnyaga (2017) designed a smart prayer mat that detects four poses likely (standing, bowing, prostration, and sitting) are done during Rakah prayer. It includes many force-sensing resistive strips inside and recognizes the place where the prayer's body is pressing. The mat detects the poses gathered from 30 participants with 100 percent. In paper (Chen, 2014) depicts a yoga pose detection framework that can detect the pose of individuals performing Yoga. Then getting the details of Yoga from the Internet reminds that individual's attention towards pose. Initially, a Kinect is utilized to capture a person's body posture and figuring out the body map. Then star skeleton, which is speed method through mapping from the centroid of the targeted object to contour extreme, can be considered a representative descriptor of human pose for yoga pose detection. Eventually, few yoga training details to detect the pose are extracted from the Internet to alert the trainer what to pay attention to when practicing the posture. This work's flow is shown in Figure 7, and the computation of star skeleton is given in Algorithm 1.

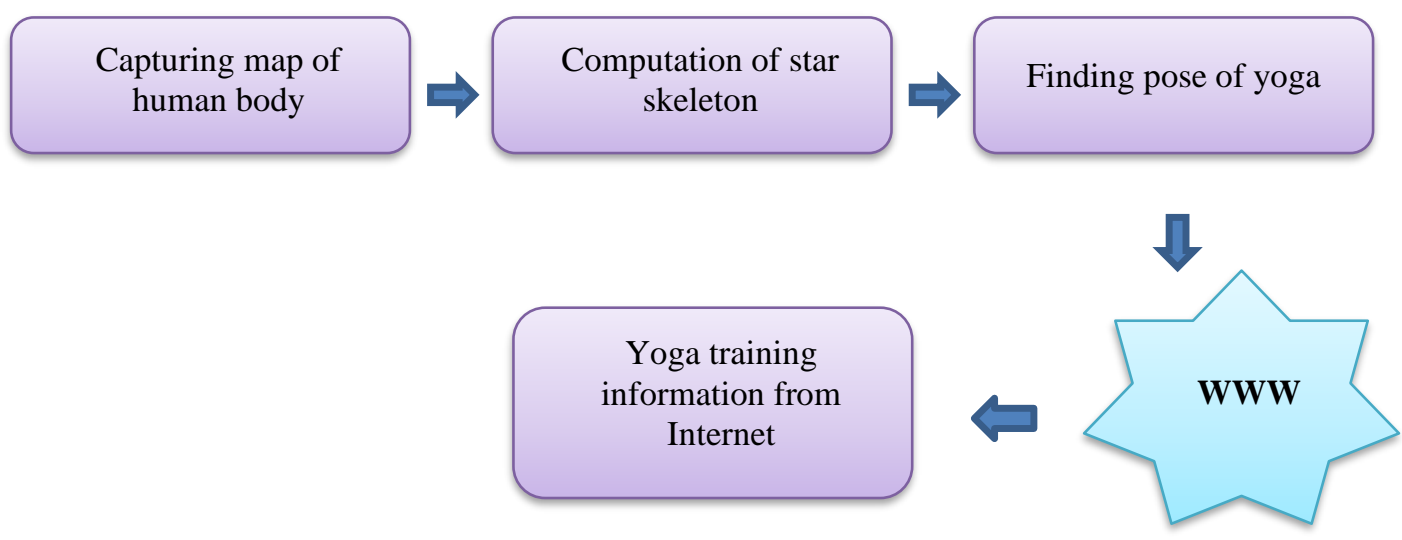

Figure 7: Flow of the training system (Chen, 2015).

In (Chinnaiah et.al, 2018), the picture is first caught utilizing a Kinect sensor that delivers a profundity picture. Figure 8 portrays the outline of the framework. The captured image may comprise various backgrounds, and to deal with the concept, a steady background produces precise results. Thus, for background deduction, these profundity pictures are prepared. From the background deducted picture, edges are recognized utilizing the canny edge identification paradigm, and this will dispose of the undesirable edges. The edge data subsequently acquired additionally prepared to use skeletonization ways for lessening closer view locales. The expert's asana is perceived in the next step, utilizing design coordinating strategies from the separated skeleton. In one part of F, the examination of asana performed concerning asana was completed knowing the expert's exactness of stance execution and, consequently, the professional assisted in rectifying a stance if required. A large dataset has been made to defeat this issue containing 5500 pictures of ten distinctive poses of yoga present and utilized a tf-present assessment paradigm that regularly points out the human body skeleton. Edges of human body joints are extricated utilizing the tf-act frame and utilized as an element to actualize different AI models. $80 \%$ of the dataset has been utilized to prepare reason, and 20 percent of the dataset is utilized while testing. This dataset is tried upon various ML grouping models and accomplishes 99.04 percent by utilizing a Random Forest Classifier. After gathering YOGA Dataset, the accompanying advances were followed, as appeared in Figure 9 (Agarwal, 2020).

In the initial phase, each image brightness is increased, and the parameter increase is set to a 2.0 for the similarity. The novel pictures were resized to $500 \times 500$ for a better fit of posture assessment calculation to obtain 
an accurate outcome. The tf-pose assessment calculation is utilized to make a skeleton of the individual playing out the Yoga represents. The measure denotes each body joint and interfaces it with a structure/stick outline like appeared in Fig 3. In the following stage, utilizing tf-present assessment calculation, the joints' directions are disengaged; the joints' quantity appears in Fig 4.

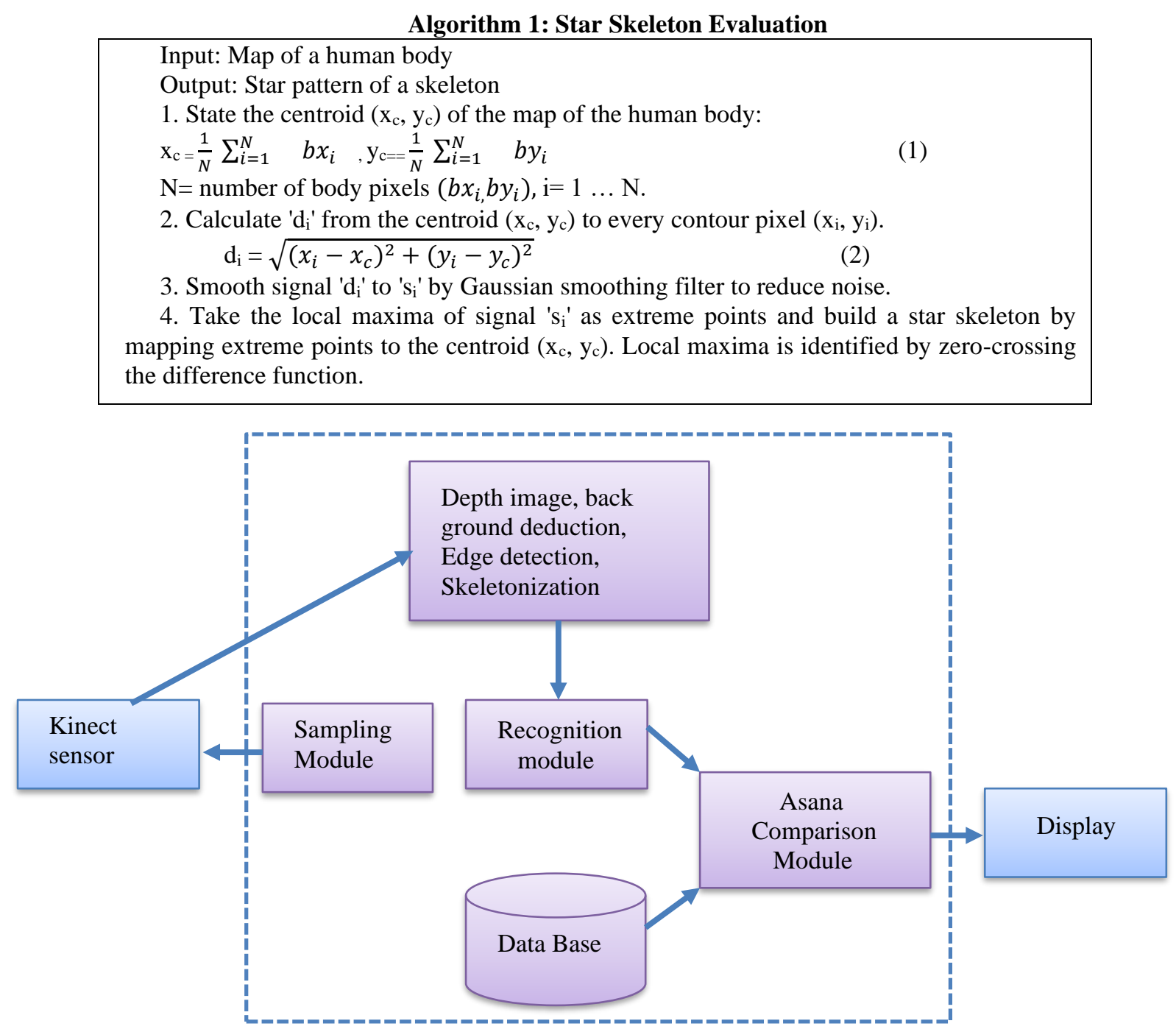

Figure 8: Embedded-based framework for Yoga

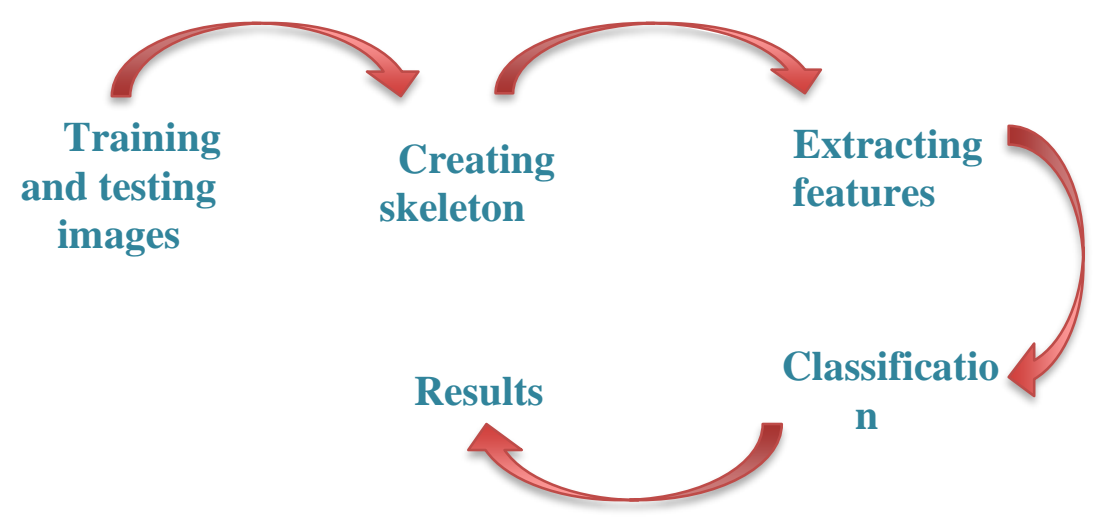

Figure 9: Flowchart of the procedure followed on the dataset 
The rules are being used to ascertain 12 distinct edges, which will be utilized as highlights to identify and address the yoga presents. The formula for figuring the point is demonstrated as given in Eq. (3).

$a^{2}=b^{2}+c^{2}-2 b c \cos A$

Where,

$\mathrm{a}, \mathrm{b}$ and $\mathrm{c}=$ distance between points $\mathrm{p} 1$ and $\mathrm{p} 2$, $\mathrm{p} 2$ and $\mathrm{p} 3$, and $\mathrm{p} 1$ and $\mathrm{p} 3$, respectively

A=angle by $\mathrm{p} 2$

If we want to evaluate the distance between any two points (Jain et.al, 2020)

$$
\mathrm{a}=\sqrt{2(X 1-X 2)+2(Y 2-Y 1)}
$$

Here, $(x 1, y 1)$ is 1 co-ordinate and $(x 2, y 2)$ is $\mathrm{p} 2$ co-ordinate.

In the last phase, the characteristics are saved in a CSV file and named accordingly. The information is later divided under training and also test datasets in an 80:20 ratio. Six classification methods of ML, such as SVM, Naive Bayes, Logistic Regression, Random Forest, KNN, and Decision Tree with distinct metrics, are utilized as a reference point to the dataset. Then final evaluations, 25 outputs of preciseness are identified. Table-I mentions the results.

Table 1: Accuracy of different classifiers over the Yoga Dataset [36].

\subsection{Evaluation Metrics}

\begin{tabular}{|l|l|}
\hline Classifier & Precision \\
\hline Logistic Regression & 0.8316 \\
\hline Random Forest & 0.9990 \\
\hline SVM & 0.9871 \\
\hline Decision Tree & 0.9761 \\
\hline Naive Bayes & 0.7475 \\
\hline KNN & 0.9891 \\
\hline
\end{tabular}

There exist numerous metrics to evaluate the accuracy of the deep learning strategies for pose estimation. The commonly used measures are the classification score, confusion matrix, and accuracy/loss curve.

Classification Score: This refers to model accuracy. It is explained as a proportion of the number of predictions to all input samples. This measurement gives excellent outcomes in a multiclass grouping when the quantity of tests in each class is nearly the equivalent.

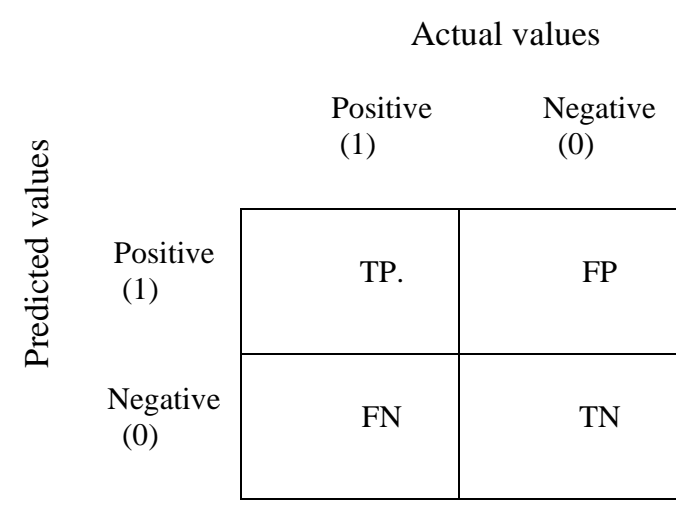

Figure 10: Sample confusion matrix

Confusion matrix: This speaks to a framework that clarifies the exactness of the model. There are four significant terms for estimating model performance. - True Positive: Predicted numeral and the actual result are both $1 . \cdot$ True Negative: Predicted digit and the real yield are both 0 . $•$ False Positive: Predicted number is one; 
however, the actual outcome is 0 . $•$ False Negative: Predicted numeral is 0 ; however, the real yield is 1 . Figure 10 shows an essential confusion matrix for paired grouping. The diagonal digits speak to the accurately ordered examples, and along these lines, we generally need the matrix diagonal to contain the highest number. If there should be an occurrence of a multiclass arrangement, every class speaks to one line and grid section.

Model accuracy and model loss curve: These are likewise considered as expectations to gather information and are generally utilized for models that adapt steadily after some interval, say, for instance, neural networks. These speak about assessment upon preparation and approval information that allows us to think about the learning model better and sum up. The model's loss curve speaks to the limiting score (loss), which implies the least score brings about better model execution. The model exactness curve speaks to an increasing score (precision), which means a higher score indicates the model's better performance. A better fitting pattern loss curve is when the training and validation loss decreases, meets the dependability state and has a negligible interval between the final loss numerals.

\section{Conclusion}

Yoga and sports have been attracting people groups for endless years; however, countless individuals receive Yoga as a significant aspect of their life from the most recent decade. Studies have been done on yoga pose identification; however, recognition of pose is still tricky because of the lack of a real-time benchmark dataset. The development of self-training systems with the current approaches, for example, using an RGB camera, is computationally expensive. Furthermore, the accuracy and robustness of Kinect-devices are also low in detecting complicated human postures. In this paper, we have presented a brief analysis of the impact of Yoga on the healthcare system in daily life. Then, human pose recognition, along with the methodologies and human body models, is described. The present methods, including conventional machine learning to advanced deep learning strategies for yoga posture recognition, are outlined along with evaluation metrics. Hence, as per the best of our knowledge, the automated systems for detecting and recognizing the postures with better performance are yet to be developed. Developing such systems with optimal performance over a maximum number of poses is considered our future work.

\section{References}

[1]. Laxman, K. (2020). Exploring the Impact of a Locally Developed Yoga Program on the Well-Being of New Zealand School-Children and Their Learning. Alternative Therapies in Health and Medicine.

[2]. Rowland, Z., \& Wenzel, M. (2020). Mindfulness and Affect-Network Density: Does Mindfulness Facilitate Disengagement from Affective Experiences in Daily Life?. Mindfulness, 1-14.

[3]. Mohan, G. (2021). Traditional Frameworks of Well-Being and Modern Science. In Handbook of Research on Evidence-Based Perspectives on the Psychophysiology of Yoga and Its Applications (pp. 410-421). IGI Global.

[4]. Wang, F., \& Boros, S. (2020). Effects of a pedometer-based walking intervention on young adults' sleep quality, stress and life satisfaction: Randomized controlled trial. Journal of Bodywork and Movement Therapies, 24(4), 286-292.

[5]. Kothari, S. (2020). Yoga Pose Classification Using Deep Learning.

[6]. Mathis, A., Schneider, S., Lauer, J., \& Mathis, M. W. (2020). A primer on motion capture with deep learning: principles, pitfalls, and perspectives. Neuron, 108(1), 44-65.

[7]. Palanimeera, J., \& Ponmozhi, K. (2020). Classification of yoga pose using machine learning techniques. Materials Today: Proceedings.

[8]. Verma, M., Kumawat, S., Nakashima, Y., \& Raman, S. (2020). Yoga-82: a new dataset for fine-grained classification of human poses. In Proceedings of the IEEE/CVF Conference on Computer Vision and Pattern Recognition Workshops (pp. 1038-1039).

[9]. Borgave, M. N. P. D. S. Role of Yoga in Battling Mental Stress during Lockdown.

[10]. Bilmez, B., \& Aral, N. (2020). Yoga for babies and children: Introduction, definition and importance of yoga, benefits of yoga, yoga in education. In Handbook of Research on Prenatal, Postnatal, and Early Childhood Development (pp. 386-397). IGI Global.

[11]. Rajender, G. A Study on Importance of Yoga in Daily Life in Telangana State.

[12]. Mala, K. Importance of Yoga and Spirituality in Modern Lifestyle.

[13]. Fourie, M., \& van der Haar, D. (2020, June). Computer Vision for the Ballet Industry: A Comparative Study of Methods for Pose Recognition. In International Conference on Business Information Systems (pp. 118-129). Springer, Cham.

[14]. Jain, S., Rustagi, A., Saurav, S., Saini, R., \& Singh, S. (2020). Three-dimensional CNN-inspired deep learning architecture for Yoga pose recognition in the real-world environment. Neural Computing and Applications, 1-15. 
[15]. Cao, Z., Simon, T., Wei, S. E., \& Sheikh, Y. (2017). Realtime multi-person 2d pose estimation using part affinity fields. In Proceedings of the IEEE conference on computer vision and pattern recognition (pp. 7291-7299).

[16]. Mehta, D., Sridhar, S., Sotnychenko, O., Rhodin, H., Shafiei, M., Seidel, H. P., ... \& Theobalt, C. (2017). Vnect: Real-time 3d human pose estimation with a single rgb camera. ACM Transactions on Graphics (TOG), 36(4), 1-14.

[17]. Wu, J., Zheng, H., Zhao, B., Li, Y., Yan, B., Liang, R., ... \& Wang, Y. (2017). Ai challenger: A largescale dataset for going deeper in image understanding. arXiv preprint arXiv: 1711.06475.

[18]. Ju, S. X., Black, M. J., \& Yacoob, Y. (1996, October). Cardboard people: A parameterized model of articulated image motion. In Proceedings of the Second International Conference on Automatic Face and Gesture Recognition (pp. 38-44). IEEE.

[19]. Chi, N. N. K. (2011). Active shape models their training and application (Doctoral dissertation, International University HCMC, Vietnam).

[20]. Loper, M., Mahmood, N., Romero, J., Pons-Moll, G., \& Black, M. J. (2015). SMPL: A skinned multiperson linear model. ACM transactions on graphics (TOG), 34(6), 1-16.

[21]. Joo, H., Simon, T., \& Sheikh, Y. (2018). Total capture: A 3d deformation model for tracking faces, hands, and bodies. In Proceedings of the IEEE conference on computer vision and pattern recognition (pp. 8320-8329).

[22]. Luvizon, D. C., Picard, D., \& Tabia, H. (2018). 2d/3d pose estimation and action recognition using multitask deep learning. In Proceedings of the IEEE Conference on Computer Vision and Pattern Recognition (pp. 5137-5146).

[23]. Martinez, J., Hossain, R., Romero, J., \& Little, J. J. (2017). A simple yet effective baseline for 3d human pose estimation. In Proceedings of the IEEE International Conference on Computer Vision (pp. 2640-2649).

[24]. Islam, M. U., Mahmud, H., Ashraf, F. B., Hossain, I., \& Hasan, M. K. (2017, December). Yoga posture recognition by detecting human joint points in real time using microsoft kinect. In 2017 IEEE Region 10 humanitarian technology conference (R10-HTC) (pp. 668-673). IEEE.

[25]. Pullen, P., \& Seffens, W. (2018). Machine learning gesture analysis of yoga for exergame development. IET Cyber-Physical Systems: Theory \& Applications, 3(2), 106-110.

[26]. Trejo, E. W., \& Yuan, P. (2018, June). Recognition of Yoga poses through an interactive system with Kinect device. In 2018 2nd International Conference on Robotics and Automation Sciences (ICRAS) (pp. 1-5). IEEE.

[27]. Chen, H. T., He, Y. Z., Chou, C. L., Lee, S. Y., Lin, B. S. P., \& Yu, J. Y. (2013, July). Computerassisted self-training system for sports exercise using kinects. In 2013 IEEE International Conference on Multimedia and Expo Workshops (ICMEW) (pp. 1-4). IEEE.

[28]. Patil, S., Pawar, A., Peshave, A., Ansari, A. N., \& Navada, A. (2011, June). Yoga tutor visualization and analysis using SURF algorithm. In 2011 IEEE Control and System Graduate Research Colloquium (pp. 43-46). IEEE.

[29]. Luo, Z., Yang, W., Ding, Z. Q., Liu, L., Chen, I. M., Yeo, S. H., ... \& Duh, H. B. L. (2011, March). "Left Arm Up!" Interactive Yoga Training in Virtual Environment. In 2011 IEEE Virtual Reality Conference (pp. 261-262). IEEE.

[30]. Wu, W., Yin, W., \& Guo, F. (2010, May). Learning and self-instruction expert system for Yoga. In 2010 2nd International Workshop on Intelligent Systems and Applications (pp. 1-4). IEEE.

[31]. Chen, H. T., He, Y. Z., Hsu, C. C., Chou, C. L., Lee, S. Y., \& Lin, B. S. P. (2014, January). Yoga posture recognition for self-training. In International Conference on Multimedia Modeling (pp. 496505). Springer, Cham.

[32]. Hsieh, C. C., Wu, B. S., \& Lee, C. C. (2011). A distance computer vision assisted yoga learning system. Journal of Computers, 6(11), 2382-2388.

[33]. Islam, M. U., Mahmud, H., Ashraf, F. B., Hossain, I., \& Hasan, M. K. (2017, December). Yoga posture recognition by detecting human joint points in real time using microsoft kinect. In 2017 IEEE Region 10 humanitarian technology conference (R10-HTC) (pp. 668-673). IEEE.

[34]. Wang, J., Huang, Z., Zhang, W., Patil, A., Patil, K., Zhu, T., ... \& Harris, T. B. (2016, December). Wearable sensor based human posture recognition. In 2016 IEEE International Conference on Big Data (Big Data) (pp. 3432-3438). IEEE.

[35]. Yao, L., Sheng, Q., Ruan, W., Gu, T., Li, X., Falkner, N., \& Yang, Z. (2015). Rf-care: Device-free posture recognition for elderly people using a passive rfid tag array.

[36]. Kasman, K., \& Moshnyaga, V. G. (2017). New technique for posture identification in smart prayer mat. Electronics, 6(3), 61 
[37]. Chen, H. T., He, Y. Z., Hsu, C. C., Chou, C. L., Lee, S. Y., \& Lin, B. S. P. (2014, January). Yoga posture recognition for self-training. In International Conference on Multimedia Modeling (pp. 496505). Springer, Cham.

[38]. Chinnaiah, M. C., Nandan, T. K., Haritha, P., Dubey, S., \& Pasha, I. A. (2018, December). A New Deliberation of Embedded based Assistive System for Yoga. In 2018 8th International Symposium on Embedded Computing and System Design (ISED) (pp. 42-47). IEEE.

[39]. Agrawal, Y., Shah, Y., \& Sharma, A. (2020, April). Implementation of Machine Learning Technique for Identification of Yoga Poses. In 2020 IEEE 9th International Conference on Communication Systems and Network Technologies (CSNT) (pp. 40-43). IEEE.

[40]. Ding, W., Hu, B., Liu, H., Wang, X., \& Huang, X. (2020). Human posture recognition based on multiple features and rule learning. International Journal of Machine Learning and Cybernetics, 11, 2529-2540.

[41]. Liu, H., Gegov, A., \& Cocea, M. (2015). Rule based systems for big data: a machine learning approach (Vol. 13). Springer.

[42]. Kumar, M. R., \& Sree, R. H. (2020). Home Computerization Monitoring System with Google Supporter. International Journal, 8(6).

[43]. Kumar, M. R., \& Gunjan, V. K. (2020). Review of machine learning models for credit scoring analysis. Ingeniería Solidaria, 16(1). 